\title{
Prevención, diagnóstico y tratamiento de la sepsis neonatal: Guía de práctica clínica basada en evidencias del Instituto Nacional Materno Perinatal del Perú
}

\author{
Prevention, diagnosis and treatment of the neonatal sepsis: Clinical practice \\ guideline-based evidence in a peruvian institute specialized
}

Carmen Dávila Aliaga ${ }^{1, a}$, Rosemary Hinojosa Pérez ${ }^{1, b}$, Elina Mendoza Ibáñez ${ }^{1, c}$, Walter Gómez Galiano ${ }^{1, d}$, Ylia Espinoza Vivas $^{1, \mathrm{e}}$, Elsa Torres Marcos ${ }^{1, \mathrm{f}}$, Carlos Velásquez Vásquez ${ }^{1, \mathrm{~g}}$, Fernando Ayque Rosas ${ }^{1, \mathrm{~h}}$, Jackelyne Alvarado Zelada ${ }^{1, \mathrm{i}}$, Gloria Corcuera Segura ${ }^{1, j}$, Nora Beltrán Gallardo ${ }^{1, \mathrm{k}}$, Josefa Gonzáles Castillo ${ }^{1,1}$, Enrique Guevara Ríos ${ }^{1, \mathrm{~m}}$, Karen Huamán Sánchez ${ }^{2,3, n}$, Celia Castillo Villacrez ${ }^{2,0}$, Nora Reyes Puma ${ }^{2,3, p}$, Patricia Caballero Ñopo ${ }^{2,3, q}$

Instituto Nacional Materno Perinatal. Lima, Perú.

${ }^{2}$ Unidad de Análisis y Generación de Evidencias en Salud Pública (UNAGESP), Instituto Nacional de Salud. Lima, Perú.

${ }^{3}$ Universidad Nacional Mayor de San Marcos. Lima, Perú.

${ }^{a}$ Médico neonatólogo, ORCID: https://orcid.org/0000-0001-9411-5703

${ }^{\mathrm{b}}$ Médico neonatólogo, ORCID: https://orcid.org/0000-0002-6640-5102

Médico neonatólogo, ORCID: https://orcid.org/0000-0001-6285-4464

${ }^{\text {d }}$ Médico neonatólogo

${ }^{\text {e}}$ Médico neonatólogo, ORCID: https://orcid.org/0000-0003-3466-7236

Médico neonatólogo, ORCID: https://orcid.org/0000-0002-2718-6621

${ }^{9}$ Médico pediatra, ORCID: https://orcid.org/0000-0001-9209-2480

${ }^{\mathrm{h}}$ Médico cirujano pediatra, ORCID: https://orcid.org/0000-0002-5508-6142

Médico cirujano pediatra, ORCID: https://orcid.org/0000-0002-8879-6642

Licenciada en enfermería, especialista en neonatologia. ORCID: https://orcid.org/0000-0003-1063-9302

${ }^{k}$ Licenciada en enfermería, especialista en neonatología.

Licenciada en enfermeria, especialista en neonatologia.

${ }^{m}$ Médico ginecoobstetra. ORCID: https://orcid.org/0000-0002-6962-2639

"Licenciada en enfermería, magister en epidemiologia. ORCID: https://orcid.org/0000-0003-3896-0421

Médico intensivista, magister en epidemiologia. ORCID: https://orcid.org/0000-0003-0588-7029

${ }^{p}$ Médico cirujano, magister en salud pública. ORCID: https://orcid.org/0000-0003-1671-5169

${ }^{9}$ Médico infectólogo, magister en salud pública. ORCID: https://orcid.org/0000-0001-9402-2531

An Fac med. 2020; 81(3):354-64. / DOI: https://doi.org/10.15381/anales.v81i3.19634.

\begin{abstract}
Correspondencia:
Karen Huamán Sánchez

khuamans@ins.gob.pe
\end{abstract}

Recibido: $1^{\circ}$ de octubre 2020 Aprobado: 18 de diciembre 2020 Publicación en línea: 30 diciembre 2020

Conflictos de interés: Los autores declaran no tener conflictos de interés.

Fuente de financiamiento: Instituto Nacional de Salud, Instituto Nacional Materno Perinatal del Perú.

Contribuciones de autoría: Todos los autores participaron en el proceso de elaboración de la guía. Karen Huamán redactó la primera versión del artículo. Todos los autores participaron en la discusión de los estudios y la formulación de las recomendaciones. Todos los autores revisaron y aprobaron la versión final del artículo

Citar como: Dávila C, Hinojosa R, Mendoza E, Gómez W, Espinoza Y, Torres $E$, et al. Prevención, diagnóstico y tratamiento de la sepsis neonatal: Guía de práctica clínica basada en evidencias del Instituto Naciona Matemo Perinatal del Perú. An Fac med. 2020;81(3):354-64. DOI: https:/doi. org/10.15381/anales.v81i3.19634.

\section{Resumen}

Introducción. La sepsis neonatal es un conjunto de signos y sintomas clínicos causados por una infección sistémica, asociada a factores de riesgo de tipo materno, neonatal u hospitalario. Objetivo. Brindar recomendaciones informadas por la mejor evidencia disponible para la prevención, diagnóstico y tratamiento de la sepsis neonatal. Métodos. Se desarrolló una guía de práctica clínica (GPC) basada en evidencias, mediante un proceso de adaptación, a cargo de un equipo de metodólogos y médicos neonatólogos expertos en el manejo clínico de la sepsis neonatal. Se realizó la búsqueda y preselección de GPC que respondan al alcance y objetivos planteados, utilizando el instrumento AGREE-II se evalúo la calidad metodológica de las guías para decidir su adaptación. Se realizó una búsqueda sistemática en múltiples bases de datos: Medline/PubMed, Embase/Ovid, Cochrane Library y LILACS, para identificar la evidencia que responda a las preguntas de la guia. Estas fueron seleccionadas y analizadas críticamente por pares clínicos y metodológicos, las recomendaciones fueron elaboradas usando el enfoque GRADE. Resultados. Se formularon 16 preguntas clínicas y recomendaciones basadas en evidencia a las que se llegó, mediante un diálogo deliberativo de expertos clínicos de diferentes hospitales de referencia para el manejo de la sepsis neonatal en el Perú. Las recomendaciones abordan la identificación de factores de riesgo, el uso de métodos confirmatorios y el tratamiento antibiótico como profilaxis y durante manejo de la enfermedad. Conclusiones: La GPC permite estandarizar el manejo clínico de la sepsis neonatal, asi como la identificación de necesidades de investigación a realizarse en el contexto peruano.

Palabras clave: Neonato; Sepsis Neonatal; Guía de Práctica Clínica; Medicina Basadaen la Evidencia; Enfoque GRADE; Perú (fuente: DeCSBIREME).

Abstract

Introduction. Neonatal sepsis is a set of clinical signs and symptoms caused by a systemic infection, associated with maternal, neonatal or hospital risk factors. Objective. Provide informed recommendations for the best available evidence for the prevention, diagnosis and treatment of neonatal sepsis. Methods an Evidence-based Clinical Practice Guide (CPG) was developed through an adaptation process, in charge of a team of methodologists and neonatologists who are experts in the clinical management of neonatal sepsis. The search and preselection of CPGs that respond to the scope and objectives set were carried out, using the AGREE-Il instrument, the methodological quality of the guides was evaluated and their adaptation decided. To identify the evidence that answers the questions in the guideline, a systematic search was carried out in multiple databases: Medline / PubMed, Embase / Ovid, Cochrane Library and LILACS. These were selected and critically analyzed by clinical and methodological peers, the recommendations were elaborated using the GRADE approach. Results. 16 clinical questions and recommendations based on evidence were formulated, which were reached, through a deliberative dialogue of clinical experts from different reference hospitals for the management of neonatal sepsis in Peru. The recommendations address the identification of risk factors, the use of confirmatory methods and antibiotic treatment as prophylaxis and during disease management. Conclusions. The CPG allows standardizing the clinical management of neonatal sepsis, as well as the identification of research needs to be carried out in the Peruvian context.

Keywords: Infant, newborn; Neonatal Sepsis; Practice Guideline; Evidence-Based Medicine; GRADE Approach; Peru (source: MeSH NLM). 


\section{INTRODUCCIÓN}

La sepsis neonatal es la segunda causa de mortalidad neonatal en el Perú $(21,77 \%, 2016)$, luego de la prematuridad (28,47\%). La mortalidad neonatal es el principal componente de la mortalidad general en todos los niños menores de 1 año $(66,6 \%)$ y de los niños menores de 5 años peruanos $(55,6 \%)^{(1,2,3)}$.

Según la Organización Mundial de la Salud (OMS), el 75\% de las muertes neonatales se presentan durante la primera semana de vida, correspondiendo al $47 \%$ del total de defunciones en niños menores de cinco años ${ }^{(4)}$. En la Región de América Latina y el Caribe, esta proporción es de $61,1 \%$ y $47,8 \%$, respectivamente ${ }^{(5)}$.

Diversos factores constituyen limitaciones para la prevención, diagnóstico y tratamiento de la sepsis neonatal, principalmente la variabilidad en múltiples aspectos como: la definición de enfermedad, un limitado conocimiento de factores de riesgo en neonatos y sus madres, la escasa información sobre la precisión de pruebas diagnósticas comúnmente utilizadas, la falta de consenso acerca del esquema de tratamiento antimicrobiano frente a la presencia de factores de riesgo materno, genera una necesidad en las instituciones de contar con mapas microbiológicos como herramienta adecuada para orientar el tratamiento ${ }^{(2,6)}$.

La sepsis neonatal es un conjunto de signos y síntomas clínicos causada por una infección sistémica, asociada a factores de riesgo maternos, neonatales $u$ hospitalarios ${ }^{(1)}$, derivado de la invasión y proliferación de bacterias, hongos o virus en el torrente sanguíneo del recién nacido (RN), manifestándose dentro de los primeros 28 días de vida, actualmente se incluye la sepsis diagnosticada después de esta edad, en recién nacidos de muy bajo peso. Se denomina sepsis temprana cuando se presenta dentro de las primeras 72 horas de vida, y sepsis tardía cuando ocurre luego de este período ${ }^{(2)}$. En la presente guía se considerarán las infecciones bacterianas como causa de sepsis ${ }^{(10,11)}$
En este contexto, esta guía de práctica clínica basada en evidencias permitirá estandarizar acciones en el diagnóstico, manejo y prevención de la sepsis neonatal, brindando recomendaciones para la toma de decisiones clínicas, que beneficien a la población y al sistema de salud peruano; por ello el Instituto Nacional Materno Perinatal (INMP) del Ministerio de Salud impulsó su realización mediante un trabajo conjunto con el Instituto $\mathrm{Na}$ cional de Salud (INS).

\section{METOdología}

Se realizó un proceso sistemático, riguroso y transparente aplicando el enfoque GRADE, siguiendo las pautas metodológicas y normativas del Ministerio de Salud del Perú ${ }^{(7)}$ (versión extensa disponible en:

https://www.inmp.gob.pe/transparencia/general/resoluciones-directorales-2019).

Conformación de los grupos y panel de expertos: El grupo elaborador (GE) de la guía estuvo integrado por expertos clínicos del INMP y metodólogos del INS, quienes brindaron asistencia técnica y transferencia tecnológica, acompañado de un panel de expertos nacionales convocado en momentos clave del proceso y en la elaboración de recomendaciones finales, conformado por neonatólogos, pediatras, ginecólogos, tecnólogos médicos, anatomopatólogos, biólogos y enfermeras representantes de hospitales de Lima y provincias, institutos sspecializados en pediatría, un centro de salud materno infantil y una sociedad científica.

Objetivo: Brindar recomendaciones informadas en evidencia para estandarizar la prevención, el diagnóstico y el manejo farmacológico oportuno de la sepsis temprana y tardía. Las recomendaciones están dirigidas a disminuir la variabilidad clínica, mejorar la calidad de atención y reducir la inequidad en la atención de RN con sepsis neonatal en el Perú.

Formulación de preguntas PICO y evaluación de guías internacionales: Se formularon en formato PICO (population, intervention, comparison y outcome, por sus siglas en inglés), a partir de los puntos de variabilidad clínica y brechas de conocimiento en el algoritmo de atención de la sepsis neonatal vigente, luego fueron priorizadas y validadas con un panel de expertos.

Para la identificación de guías, se consultaron bases de datos y repositorios oficiales de instituciones internacionales desarrolladoras de guías, identificándose siete guías, dos se excluyeron por encontrarse en idioma alemán, cuatro fueron eliminadas después de aplicar criterios de preselección (rigurosidad de la guía, año de publicación, similitud con la población objetivo y coincidencia con el tópico de estudio). Se evaluó la calidad utilizando el instrumento AGREE II (http:// www.agreetrust.org)(8), solo una guía, "Guía de práctica clínica. Recién nacido: sepsis neonatal temprana" (Colombia) obtuvo un puntaje de $87 \%$ en evaluación global y mayores al $80 \%$ en dominios críticos (alcance y objetivos, rigor en la elaboración y aplicabilidad) ${ }^{(9)}$.

Proceso de revisión y síntesis de la evidencia: Mediante una matriz se compararon las preguntas clínicas de la guía seleccionada con las generadas por el GE, para definir las posibles a adaptar y las nuevas o de "novo". Para las preguntas adaptadas, se actualizaron las estrategias de búsqueda sistemática para incluir nueva evidencia disponible; mientras que para las preguntas de "novo" se construyeron nuevas estrategias en las bases de datos de Medline (PubMed), Embase (Ovid), Cochrane Library y LILACs (BvS OPS). La selección de evidencias se realizó mediante pares del INMP y el INS de acuerdo con criterios de inclusión y exclusión preestablecidos, y según jerarquía de evidencia, iniciando con revisiones sistemáticas con o sin metaanálisis, ensayos clínicos aleatorizados y estudios observacionales.

Gradación del cuerpo de la evidencia mediante GRADE: Fue categorizada en cuatro niveles: alta, moderada, baja y muy baja (Tabla 1). Para cada pregunta se elaboraron tablas de resumen de hallazgos GRADE (Summary of Findings tables/SoF) ${ }^{(10)}$. 
Tabla 1. Interpretación de los niveles de certeza de la evidencia, según la metodologia de Grading of Recommendations Assessment, Development, and Evaluation (GRADE).

\section{Nivel de evidencia GRADE}

Alta

\begin{tabular}{ll}
\hline Moderada & la certeza de los resultados. \\
\hline Baja & $\begin{array}{l}\text { Probablemente puedan existir nuevos estudios que influyan } \\
\text { en la confianza de los resultados estimados y puedan } \\
\text { modificarlo. }\end{array}$ \\
\hline Muy baja & $\begin{array}{l}\text { Es muy probable que nuevos estudios influyan en la confianza } \\
\text { de los resultados estimados y los modifiquen. }\end{array}$ \\
\hline
\end{tabular}

\section{RECOMENDACIONES}

Formulación de las recomendaciones y diálogo deliberativo

Se construyeron marcos de "evidencia para la decisión" (Evidence to decisions/ EtD) utilizando la plataforma virtual GRADEpro (https://gradepro.org/), considerando los beneficios y daños potenciales, valores y preferencias, aceptabilidad y factibilidad de la intervención, así como el uso de recursos necesarios, para determinar la fuerza y dirección de las recomendaciones (Tabla 2), mediante un diálogo deliberativo, en el que participó el panel de expertos clínicos y el GE de la guía ${ }^{(11)}$. Finalmentemente, la presente guía tiene 16 preguntas clínicas y recomendaciones informadas en evidencia (tabla 3 y 4 ).

\section{PREVENCIÓN}

Pregunta 1. ¿En recién nacidos (RN) hijos de madres con ruptura prematura de membranas (RPM) $>18$ horas que recibieron tratamiento antibiótico, disminuye el

Tabla 2. Interpretación de la fuerza de la recomendación, según la metodología de Grading of Recommendations Assessment, Development, and Evaluation (GRADE).

\begin{tabular}{ll}
$\begin{array}{l}\text { Fuerza de la } \\
\text { recomendación }\end{array}$ & \multicolumn{1}{c}{ Significado } \\
\hline Fuerte a favor & $\begin{array}{l}\text { Los efectos deseables superan los efectos indeseables. } \\
\text { Se recomienda hacerlo }\end{array}$ \\
\hline Condicional a favor & $\begin{array}{l}\text { Los efectos deseables probablemente superan los efectos indeseables. } \\
\text { Se sugiere hacerlo }\end{array}$ \\
\hline Condicional en contra & $\begin{array}{l}\text { Los efectos indeseables probablemente superan los efectos deseables. } \\
\text { Se sugiere no hacerlo }\end{array}$ \\
\hline Fuerte en contra & $\begin{array}{l}\text { Los efectos indeseables superan los efectos deseables. } \\
\text { Se recomienda no hacerlo }\end{array}$ \\
\hline
\end{tabular}

lo tanto, es necesario evitar el uso de antibióticos en hijos asintomáticos de madres con RPM > de 18 horas como único factor de riesgo.

La implementación de esta recomendación generará ahorro de recursos derivados de la disminución del uso no controlado de antibióticos y la reducción de la hospitalización.

Pregunta 2. ¿En RN hijos de madres que presentan corioamnionitis (definida solamente con criterios clínicos), el inicio de tratamiento antibiótico en el período neonatal inmediato disminuye el riesgo de infección neonatal y la morbilidad?

\section{Recomendación}

La evidencia señala que el antecedente materno de corioamnionitis incrementa el riesgo de sepsis neonatal y leucomalacia periventricular en $\mathrm{RN}$ con $<34$ semanas de edad gestacional ${ }^{(14)}$.

Se recomienda el uso de tratamiento antibiótico en RN pretérmino hijos de madre con corioamnionitis. Recomendación condicional a favor de la intervención, basada en baja calidad de la evidencia.

Se recomienda el uso de tratamiento antibiótico en todos los RN hijos de madre con corioamnionitis. Recomendación condicional a favor de la intervención, basada en consenso de expertos.

\section{De la evidencia científica a la práctica clínica}

Existen grandes beneficios en el tratamiento del recién nacido de madre con antecedente de corioamnionitis clínica materna, sobre todo en prematuros y en el riesgo de leucomalacia periventricular. Los daños potenciales de iniciar el tratamiento en este grupo están relacionados al uso de la terapia antibiótica.

Pregunta 3. En RN, hijos de madres con fiebre $>38^{\circ} \mathrm{C}$ (no relacionada con corioamnionitis), ¿la iniciación de tratamiento antibiótico en el periodo neonatal inmediato disminuye el riesgo de infección neonatal y mortalidad? Recomendación

La búsqueda de evidencias no reportó artículos que soporten que el uso de 
Tabla 3. Preguntas PICO de la guía de práctica clínica informada en evidencias de sepsis neonatal.

\section{Tema} $\mathbf{N}^{\circ}$ Pregunta

¿En recién nacidos (RN), hijos de madres con Ruptura Prematura de Membranas (RPM) $>18$ horas, el uso de

PICO 1 tratamiento antibiótico disminuye el riesgo de infección neonatal y mortalidad comparado con aquellos que no recibieron tratamiento antibiótico?

En RN, hijos de madres que presentan corioamnionitis PICO 2 (definida solamente con criterios clínicos), cel inicio de Prevención disminuye el riesgo de infección neonatal y morbilidad?

En RN, hijos de madres con fiebre $>38^{\circ} \mathrm{C}$ (no relacionada con

PICO 3 corioamnionitis), ¿̇la iniciación de tratamiento antibiótico en el periodo neonatal inmediato disminuye el riesgo de infección neonatal y mortalidad?

\begin{tabular}{ll}
\hline PICO 4 & $\begin{array}{l}\text { ¿Qué factores de riesgo materno y fetal se asocian con sepsis } \\
\text { neonatal para orientar el manejo? }\end{array}$ \\
\hline
\end{tabular}

En RN con factores de riesgo para sepsis neonatal temprana,

PICO 5 ¿cuántos hallazgos al examen físico tienen buena sensibilidad y especificidad para detectar infección neonatal?

En RN con sospecha de sepsis neonatal, ¿el hemograma y

PICO 6 las medidas derivadas del mismo como índices leucocitarios, tienen alta sensibilidad para detectar infección neonatal?

Diagnóstico En RN con sospecha de sepsis neonatal, atendidos en

PICO 7 sitios donde esté disponible, ¿la procalcitonina tiene alta sensibilidad para detectar infección neonatal?

En RN con sospecha de infección neonatal, clas escalas

PICO 8 sumativas de reportes de laboratorio de PCR y hallazgos del hemograma tienen buenas características operativas para detectar infección neonatal?

En RN con sepsis probable, ¿̇la toma de 2 o más hemocultivos

PICO 9 incrementa la sensibilidad versus la toma de 1 hemocultivo, para el diagnóstico?

En RN con sospecha de infección neonatal temprana, ¿cuáles

PICO 10 son los factores de riesgo asociados a una probabilidad de neumonía lo suficientemente alta para que se justifique

Diagnóstico

confirmatorio tomar una radiografía de tórax?

PICO 11 ¿La toma rutinaria de urocultivo debe formar parte del estudio confirmatorio de sepsis neonatal temprana?

En RN con sospecha de infección neonatal temprana, ¿cuáles

PICO 12 son los factores de riesgo asociados a una probabilidad de meningitis lo suficientemente alta para que se justifique una punción lumbar?

En RN con criterios para iniciar tratamiento para sepsis

PICO 13 neonatal temprana, ¿qué esquemas antibióticos son adecuados en términos de efectividad y seguridad?

\begin{tabular}{ll} 
adecuados en términos de efectividad y seguridad? \\
\hline PICO 14 & $\begin{array}{l}\text { En RN con sospecha de infección neonatal con resultado } \\
\text { de hemocultivos negativos, ¿cuál es la duración óptima del } \\
\text { tratamiento antibiótico? }\end{array}$ \\
\hline
\end{tabular}

Tratamiento

En RN con sospecha de infección neonatal con resultado

PICO 15 de hemocultivos positivos, ¿cuál es la duración óptima del tratamiento antibiótico?

PICO 16

¿Cuál es el régimen de tratamiento antibiótico óptimo para la meningitis neonatal? antibióticos disminuye el riesgo de morbimortalidad en RN hijos de madre con fiebre $>38^{\circ} \mathrm{C}$ como condición aislada.

En RN hijos de madres con fiebre $>38$ ${ }^{\circ} \mathrm{C}$ como condición aislada no se recomienda el uso de antibióticos de rutina. Recomendación fuerte en contra de la intervención, basada en muy baja calidad de la evidencia.

\section{De la evidencia científica a la práctica clínica}

No existen beneficios acerca del uso de antibióticos de rutina en RN hijos de madres con fiebre $>38^{\circ} \mathrm{C}$ como condición aislada durante al trabajo de parto. No hay diferencias en el desenlace de morbimortalidad. Por otro lado, los daños potenciales del uso de antibióticos son la resistencia bacteriana y la estancia hospitalaria prolongada innecesariamente ${ }^{(15,16,17)}$.

Pregunta 4. ¿Qué otros factores de riesgo materno y fetal se asocian con sepsis neonatal para orientar el manejo?

\section{Recomendación}

La evidencia revisada fue de baja calidad, pero identifica como factores de riesgo para sepsis temprana a la adolescencia materna $<15$ años, RPM $>18$ h, fiebre materna $>38^{\circ} \mathrm{C}$, ITU y corioamnionitis, así como la prematuridad.

Se recomienda tomar en consideración los factores de riesgo como adolescencia materna $<15$ años, fiebre materna $>38^{\circ} \mathrm{C}$, ITU y prematuridad, previo al inicio del protocolo diagnóstico de sepsis neonatal. Recomendación basada en evidencia de baja calidad.

Adicionalmente se recomienda considerar la vulvovaginitis como otro factor de riesgo de sepsis neonatal. Recomendación basada en consenso de expertos.

\section{De la evidencia científica a la práctica clínica}

Existe un gran potencial para reducir las infecciones neonatales de inicio temprano centrando las intervenciones en mujeres con infecciones maternas (signos clínicos confirmados por laboratorio), colonización y factores de riesgo de infección (RPM, la ruptura de membrana y la ruptura prolongada de membrana) ${ }^{(18,19)}$. Es importante comprender la etiología de las infecciones
Fuente: Guía de práctica clínica para la prevención, diagnóstico y tratamiento de la sepsis neonatal. Versión extensa- Instituto Nacional Materno Perinatal. RD N²90-2019-DG-INMP/MINSA. 
Tabla 4. Recomendaciones de la guía de práctica clínica informada en evidencias de sepsis neonatal.

\section{Tema}

政

-

PICO 1 No existe evidencia de que el uso sistemático de antibióticos en RN, hijos de madres con RPM >18h como único factor de riesgo modifique la probabilidad
de infección y mortalidad del recién nacido. El panel recomienda no administrar antibióticos de rutina al RN hijo de madre con RPM $>18 \mathrm{~h}$ como único factor de riesgo. Recomendación fuerte en contra de la intervención, basada en muy baja calidad de la evidencia.

El panel recomienda no administrar antibióticos de rutina al RN hijo de madre con RPM $>18 \mathrm{~h}$ como único factor de riesgo.
Recomendación fuerte en contra de la intervención, basada en muy baja calidad de la evidencia.

Prevención Recomendación condicional a favor de la intervención, basada en baja calidad de la evidencia.

PICO 3 En RN hijos de madres con fiebre $>38^{\circ} \mathrm{C}$ como condición aislada no se recomienda el uso de antibióticos de rutina.

PICO 3 Recomendación fuerte en contra de la intervención, basada en muy baja calidad de la evidencia.

La evidencia revisada es de baja calidad, pero identifica como factores de riesgo para sepsis temprana: adolescencia $<15$ años, ruptura prematura de membranas $>18$ h, prematuridad, fiebre materna $>38^{\circ} \mathrm{C}$, ITU y corioamnionitis. PICO 4 Se recomienda tomar en consideración los factores de riesgo como adolescencia $<15$ años, prematuridad, fiebre materna $>38^{\circ} \mathrm{C}$ e ITU previo al inicio del

Recomendación basada en evidencia de baja calidad.

Adicionalmente se recomienda considerar la vulvovaginitis como otro factor de riesgo de sepsis neonatal. Recomendación basada en consenso de expertos.

\begin{tabular}{|c|c|c|}
\hline \multirow{4}{*}{ Diagnóstico } & PICO 5 & $\begin{array}{l}\text { La evidencia revisada no aporta parámetros clínicos para evaluar sensibilidad y especificidad en la determinación de sepsis temprana, y reporta que la } \\
\text { presencia de apnea, hipotermia y letargia como signos clínicos tienen mayor especificidad para sepsis tardía en prematuros. } \\
\text { En RN con factores de riesgo para sepsis se recomienda considerar como criterios la presencia de tres o más signos clínicos como: taquicardia, apnea, } \\
\text { hipotermia y letargia, que ameriten la toma de cultivos de laboratorio y el uso de antibióticos. } \\
\text { Recomendación fuerte a favor de la intervención, basada en baja calidad de la evidencia. }\end{array}$ \\
\hline & PICO 6 & $\begin{array}{l}\text { No se encontró evidencia directa que responda a la pregunta; sin embargo, un estudio de tipo cohorte reportó que existe mayor especificidad de la prueba } \\
\text { (hemograma completo: recuento leucocitario y neutrófilos totales) cuando se toma a partir de las } 4 \text { horas. } \\
\text { Se sugiere utilizar el recuento leucocitario, neutrófilos totales, relación inmaduros/ neutrófilos totales en recién nacidos con sospecha de sepsis neonatal } \\
\text { para diagnóstico. } \\
\text { Recomendación fuerte a favor de la intervención basada en moderada calidad de la evidencia. }\end{array}$ \\
\hline & PICO 7 & $\begin{array}{l}\text { La evidencia reporta una alta sensibilidad y especificidad de la prueba. } \\
\text { Se recomienda la procalcitonina para el diagnóstico temprano de sepsis neonatal tardía como prueba coadyuvante de alta especificidad. } \\
\text { Recomendación fuerte a favor de la intervención, basada en moderada calidad de la evidencia. }\end{array}$ \\
\hline & PICO 8 & $\begin{array}{l}\text { La evidencia en relación con las escalas sumativas reporta una alta especificidad para el diagnóstico de sepsis temprana. } \\
\text { Se recomienda la prueba de PCR cuantitativa en el diagnóstico y manejo de la sepsis neonatal, siendo la prueba de mayor valor en el monitoreo del } \\
\text { tratamiento. } \\
\text { Recomendación fuerte a favor de la intervención basada en moderada calidad de la evidencia. }\end{array}$ \\
\hline \multirow{4}{*}{$\begin{array}{l}\text { Diagnóstico } \\
\text { confirmatorio }\end{array}$} & PICO 9 & $\begin{array}{l}\text { No se encontró evidencia para responder la pregunta. } \\
\text { El panel de expertos recomienda la realización de } 2 \text { hemocultivos en puntos venosos periféricos diferentes, en neonatos con sepsis probable, previo al } \\
\text { inicio de tratamiento antibiótico. } \\
\text { Recomendación fuerte a favor de la intervención, basada en opinión de expertos. }\end{array}$ \\
\hline & PICO 10 & $\begin{array}{l}\text { Se recomienda no tomar radiografías de tórax de manera rutinaria en RN con sospecha de sepsis neonatal temprana si no presentan síntomas respiratorios. } \\
\text { Recomendación fuerte en contra de la intervención. } \\
\text { Se recomienda tomar radiografías de tórax, a criterio médico, en aquellos pacientes que presenten signos o síntomas respiratorios (taquipnea, FR }>60 \text { por minuto } \\
\text { persistente), retracciones intercostales, subcostales, supraclaviculares; cianosis; desaturación evidente en oximetría; hallazgos auscultatorios (estertores, etc.). } \\
\text { Recomendación fuerte a favor de la intervención basada en muy baja calidad de la evidencia. }\end{array}$ \\
\hline & PICO 11 & $\begin{array}{l}\text { La evidencia muestra que la incidencia de infección urinaria y de urocultivos positivos, como parte del diagnóstico de sepsis neonatal temprana es } \\
\text { extremadamente baja. Un resultado negativo de urocultivo no ayuda a confirmar o descartar sepsis temprana (baja sensibilidad). En las infrecuentes } \\
\text { ocasiones en que es positivo, sirve para confirmar el diagnóstico (evidencia de baja calidad). } \\
\text { Se recomienda no tomar urocultivo de rutina como parte del estudio del RN con sospecha de sepsis neonatal temprana. } \\
\text { Recomendación fuerte en contra de la intervención, basada en baja calidad de la evidencia. }\end{array}$ \\
\hline & PICO 12 & $\begin{array}{l}\text { Se encontró evidencia de baja calidad que reporta baja incidencia de meningitis asociada a sepsis neonatal temprana. Asimismo no existen diferencias } \\
\text { significativas en la comparación de grupos a los que se realiza y no se realiza punción lumbar (PL) antes de las } 72 \text { horas. } \\
\text { No se recomienda rutinariamente realizar PL en recién nacidos con sospecha de sepsis neonatal temprana. } \\
\text { Recomendación fuerte en contra de la intervención basada en muy baja calidad de la evidencia. }\end{array}$ \\
\hline \multirow{4}{*}{ Tratamiento } & PICO 13 & $\begin{array}{l}\text { Existe evidencia de baja calidad acerca de que el uso del esquema de ampicilina más aminoglucósido como primera línea cuente con un perfil de efectividad } \\
\text { y seguridad adecuado; de igual manera no se encontró diferencia con el uso del esquema de penicilina más aminoglucósido. } \\
\text { Se recomienda como esquema de tratamiento inicial en recién nacidos tanto pretérmino como a término para infección neonatal temprana la combinación } \\
\text { de ampicilina y gentamicina. Se sugiere monitorizar las tasas de fracaso terapéutico y los perfiles de sensibilidad a los antibióticos en los gérmenes } \\
\text { identificados en cada institución, para guiar de forma más precisa la selección de esquemas antibióticos para sepsis temprana y tardía. } \\
\text { Recomendación fuerte a favor de la intervención basada en muy baja calidad de la evidencia. }\end{array}$ \\
\hline & PICO 14 & $\begin{array}{l}\text { En RN con sospecha de sepsis, con hemocultivos negativos y con evolución clínica favorable, se recomienda suspender el tratamiento antibiótico entre las } 48 \text { y } 72 \text { horas. } \\
\text { Recomendación fuerte a favor de la intervención basada en opinión de expertos. } \\
\text { En RN con sospecha de sepsis, con hemocultivos negativos y clínica sospechosa de infección (o sin evolución clínica favorable), se recomienda continuar el tratamiento } \\
\text { antibiótico por } 7 \text { días. } \\
\text { Recomendación fuerte a favor de la intervención basada en opinión de expertos. } \\
\text { En caso de pobre respuesta ofracaso terapéutico, debe pasar a segunda línea de antibiótico, según el mapa microbiológico institucional. } \\
\text { Recomendación fuerte a favor de la intervención basada en opinión de expertos. }\end{array}$ \\
\hline & PICO 15 & $\begin{array}{l}\text { En RN con hemocultivos positivos y con evolución clínica favorable, se recomienda completar } 7 \text { días de tratamiento antibiótico. } \\
\text { Recomendación fuerte a favor de la intervención basada en baja calidad de la evidencia. } \\
\text { En caso de pobre respuesta o fracaso terapéutico, debe pasar a segunda línea de antibiótico, según mapa microbiológico institucional. } \\
\text { Recomendación fuerte a favor de la intervención basada en muy baja calidad de la evidencia }\end{array}$ \\
\hline & PICO 16 & $\begin{array}{l}\text { Se recomienda dar tratamiento antibiótico por al menos } 14 \text { días para meningitis neonatal, evaluando la respuesta clínica, características bioquímicas y } \\
\text { microbiológicas del LCR y estudio de imágenes cerebral. } \\
\text { Recomendación fuerte a favor de la intervención basada en consenso de expertos. } \\
\text { Se recomienda determinar el régimen antibiótico de acuerdo al mapa microbiológico de la institución en las meningitis asociadas a sepsis tardía. En las } \\
\text { meningitis asociadas a sepsis temprana o adquirida en la comunidad se recomienda iniciar tratamiento con ampicilina y cefotaxima a dosis que logren } \\
\text { penetrabilidad en el LCR. } \\
\text { Recomendación fuerte a favor de la intervención basada en consenso de expertos. }\end{array}$ \\
\hline
\end{tabular}

Fuente: Guía de práctica clínica para la prevención, diagnóstico y tratamiento de la sepsis neonatal. Versión extensa- Instituto Nacional Materno Perinatal. RD N²90-2019-DG-INMP/MINSA. 
maternas, su colonización y las infecciones neonatales en los países de ingresos bajos y medianos. La estandarización de definiciones para infecciones maternas y recién nacidos sería útil para comparar estudios.

Los daños potenciales de iniciar el tratamiento antibiótico en recién nacidos hijos de madres con los factores de riesgo descritos están relacionados a las reacciones adversas de la terapia antibiótica.

Pregunta 5. En RN con factores de riesgo para sepsis neonatal temprana, ¿cuáles son los hallazgos al examen físico que tienen buena sensibilidad y especificidad para detectar infección neonatal? Recomendación

No se encontró evidencia que responda directamente a la pregunta; sin embargo, se encontró un estudio que reporta: apnea, hipotermia y letargia como signos clínicos de mayor especificidad para sepsis tardía en prematuros.

En RN con factores de riesgo para sepsis se recomienda considerar como criterios clínicos la presencia de 3 o más signos clínicos que ameriten la toma de cultivos de laboratorio y el uso de antibióticos. Recomendación fuerte a favor de la intervención, basada en baja calidad de la evidencia.

\section{De la evidencia a la práctica clínica}

Los beneficios de tomar en cuenta indicadores clínicos, en la evaluación de RN prematuros de muy bajo peso con sospecha de sepsis neonatal, permitiría mejorar la posibilidad estadística de diagnóstico y tratamiento de sepsis neonatal tardía, sobre todo si combinamos los hallazgos de indicadores clínicos como apnea, hipotermia y letargia (20).

Los daños potenciales estarían relacionados a la toma de exámenes de laboratorio e inherentes al tratamiento, así como a los costos de ambos en el caso que no se confirmara sepsis neonatal tardía.

Pregunta 6. ¿En RN con sospecha de infección neonatal el hemograma y las medidas derivadas del mismo como índices leucocitarios, tienen alta sensibilidad para detectar infección neonatal?

\section{Recomendación}

No se encontró evidencia directa que responda a la pregunta; sin embargo, un estudio de tipo cohorte reportó que existe mayor sensibilidad y especificidad cuando se toma la prueba a partir de 4 horas.

Se sugiere utilizar el recuento leucocitario, neutrófilos totales, relación inmaduros/ neutrófilos totales a los RN con sospecha de sepsis neonatal para diagnóstico.

\section{De la evidencia científica a la práctica clínica}

Los marcadores hemáticos constituyen herramientas predictivas para el diagnóstico oportuno de sepsis neonatal, considerando el riesgo de diagnósticos erróneos debido a falsos positivos que generan un uso innecesario de antibióticos y hospitalización (21,22). La factibilidad de la implementación de esta recomendación dependerá del entrenamiento del personal a cargo del procesamiento de muestras y la disposición de sistemas automatizados e insumos necesarios.

Pregunta 7. ¿En RN con sospecha de infección neonatal, atendidos en sitios donde se encuentre disponible, la procalcitonina tiene alta sensibilidad para detectar infección neonatal?

\section{Recomendación}

La evidencia reporta una alta sensibilidad y especificidad de la prueba.

Se recomienda la procalcitonina para el diagnóstico temprano de sepsis neonatal como prueba coadyuvante de alta especificidad. Recomendación fuerte a favor de la intervención, basada en moderada calidad de la evidencia.

\section{De la evidencia científica a la práctica clínica}

Realizar la prueba de procalcitonina en RN con sospecha de sepsis temprana representa un beneficio sustancial en el diagnóstico e inicio de tratamiento oportuno $(23,24,25)$

Sin embargo, la factibilidad de agregar el uso rutinario de procalcitonina dentro de las pruebas requeridas para el diagnóstico de sepsis neonatal, se relacionan a la disponibilidad de la prueba y los costos relacionados.
Pregunta 8. ¿En RN con sospecha de infección neonatal, las escalas sumativas de reportes de laboratorio de proteína $C$ reactiva (PCR) y hallazgos del hemograma tienen buenas características operativas para detectar infección neonatal?

\section{Recomendación}

La evidencia reporta para las escalas sumativas de pruebas de laboratorio una baja sensibilidad, pero alta especificidad para el diagnóstico de sepsis temprana.

Se recomienda la prueba de PCR cuantitativa en el diagnóstico y manejo de la sepsis neonatal, siendo la prueba de mayor valor en el monitoreo del tratamiento. Recomendación fuerte a favor de la intervención basada en moderada calidad de la evidencia.

\section{De la evidencia científica a la práctica clínica}

Los beneficios del uso de escalas sumativas de reportes de laboratorio de PCR y hallazgos del hemograma se encuentran relacionados con el diagnóstico temprano de sepsis neonatal y el monitoreo de la eficacia del tratamiento ${ }^{(25,26)}$. Dentro de los daños potenciales de la intervención podría considerarse el dolor por la punción durante la toma de la muestra.

Pregunta 9. ¿En RN con sepsis probable, la toma de 2 o más hemocultivos incrementa la sensibilidad versus la toma de 1 hemocultivo, para el diagnóstico?

\section{Recomendación}

No se encontró evidencia de buena calidad para responder directamente a la pregunta (27).

Se recomienda la realización de 2 hemocultivos en puntos venosos periféricos diferentes, en neonatos con sepsis probable, previo al inicio de tratamiento antibiótico. Recomendación fuerte a favor de la intervención, basada en opinión de expertos.

\section{De la evidencia científica a la práctica} clínica

Los beneficios de la realización de 2 hemocultivos en puntos venosos periféricos diferentes, en RN con sepsis probable, 
previo al inicio de tratamiento antibiótico, podría permitir con mayor posibilidad la identificación del agente patógeno causal de la sepsis, además permite minimizar los falsos positivos, al identificar un agente contaminante. Los daños potenciales de usar dos hemocultivos se relacionan al mayor volumen requerido de sangre del paciente para la segunda muestra, además de los costos inherentes.

Pregunta 10. En RN con sospecha de sepsis neonatal temprana, ¿cuáles son los factores de riesgo asociados a una probabilidad de neumonía lo suficientemente alta para que se justifique tomar una radiografía de tórax?

\section{Recomendación}

Se recomienda no tomar radiografías de tórax de manera rutinaria en RN con sospecha de sepsis neonatal temprana si no presentan síntomas respiratorios. Recomendación fuerte en contra de la intervención.

Se recomienda tomar radiografías de tórax, a criterio médico, en aquellos pacientes que presenten signos o síntomas respiratorios (taquipnea, FR $>60$ por minuto persistente), retracciones intercostales, subcostales, supraclaviculares; cianosis; desaturación evidente en oximetría; hallazgos auscultatorios (estertores, etc.). Recomendación fuerte a favor de la intervención basada en muy baja calidad de la evidencia.

\section{De la evidencia científica a la práctica clínica}

Basada en evidencia indirecta identificada durante la búsqueda ${ }^{(28)}$, los daños potenciales estarían ocasionados por la exposición de los recién nacidos a los rayos $x$, además de la elevación de los costos de atención. El panel de expertos consideró que existen mayores beneficios al evitar la exposición innecesaria a la radiación, en $\mathrm{RN}$ que no presentan signos respiratorios. Es importante monitorizar permanentemente los signos de alarma en RN con factores de riesgo.

Pregunta 11. ¿La toma rutinaria de urocultivo debe formar parte del estudio confirmatorio de sepsis neonatal temprana?

\section{Recomendación}

La evidencia muestra que la incidencia de infección urinaria y de urocultivo posi- tivo, como parte del diagnóstico de sepsis neonatal temprana es extremadamente baja. Un resultado negativo de urocultivo no ayuda a confirmar o descartar sepsis temprana (baja sensibilidad). En las infrecuentes ocasiones en que es positivo, sirve para confirmar el diagnóstico.

Se recomienda no tomar urocultivo de rutina como parte del estudio del RN con sospecha de sepsis neonatal temprana. Recomendación fuerte en contra de la intervención, basada en baja calidad de la evidencia.

\section{De la evidencia científica a la práctica clínica}

Realizar un urocultivo de rutina implica procedimientos invasivos como la inserción de un catéter vesical o la punción suprapúbica, considerados innecesarios, además de ser una fuente de exposición a la posibilidad de infecciones recurrentes ${ }^{(29)}$.

Pregunta 12. En RN con sospecha de infección neonatal temprana, ¿cuáles son los factores de riesgo asociados a una probabilidad de meningitis lo suficientemente alta para que se justifique una punción lumbar?

\section{Recomendación}

Se encontró evidencia de baja calidad que reporta baja incidencia de meningitis asociada a sepsis neonatal temprana. Asimismo, no existen diferencias significativas en la comparación de grupos a los que se realiza y no se realiza punción lumbar (PL) antes de las 72 horas ${ }^{(30-35)}$.

No se recomienda rutinariamente realizar PL en recién nacidos con sospecha de sepsis neonatal temprana. Recomendación fuerte en contra de la intervención basada en muy baja calidad de la evidencia.

De la evidencia científica a la práctica clínica

Los beneficios de realizar PL en RN con sospecha de sepsis son muy pocos, mientras que los daños potenciales son altos debido al riesgo que presentan todos los procedimientos invasivos en el RN. El balance entre las consecuencias favorables y desfavorables favorece evitar la PL en
RN con sospecha de sepsis y sin cuadro neurológico presente.

Pregunta 13. En RN con criterios para iniciar tratamiento para sepsis neonatal temprana, ¿qué esquemas antibióticos son adecuados en términos de efectividad y seguridad?

\section{Recomendación}

Existe evidencia de baja calidad acerca de que el uso del esquema de ampicilina más aminoglucósido como primera línea cuente con un perfil de efectividad y seguridad adecuado; de igual manera, no se encontró diferencia con el uso del esquema de penicilina más aminoglucósido.

Se recomienda como esquema de tratamiento inicial en recién nacidos tanto pretérmino como a término para sepsis neonatal temprana la combinación de ampicilina y gentamicina. Se sugiere monitorizar las tasas de fracaso terapéutico y los perfiles de sensibilidad a los antibióticos en los gérmenes identificados en cada institución, para guiar de forma más precisa la selección de esquemas antibióticos para sepsis temprana y tardía. Recomendación fuerte a favor de la intervención basada en muy baja calidad de la evidencia.

\section{De la evidencia científica a la práctica clínica}

Los beneficios del uso de la combinación de ampicilina y aminoglucósido son grandes debido a que el espectro de gérmenes tomados por esta combinación es amplio y es aceptada por la comunidad médica ${ }^{(36)}$. Los daños son mínimos ya que no existen reportes de efectos adversos por el uso de la combinación de antibióticos en el manejo de la sepsis temprana en neonatología. Es factible la implementación de esta intervención ya que son medicamentos accesibles.

Pregunta 14. En RN con sospecha de infección neonatal con resultado de hemocultivos negativos, ¿cuál es la duración óptima del tratamiento antibiótico? Recomendación

En RN con sospecha de infección, con hemocultivos negativos y con evolución 
Tabla 5. Necesidades de investigación en el manejo de sepsis neonatal.

- Uso de lactato como marcador de complicaciones en sepsis neonatal.

- Asociación de coagulación intravascular diseminada con sepsis neonatal y shock séptico.

- Determinación de agentes etiológicos prevalentes en meningitis asociadas a sepsis tardía en neonato en el Perú.

- Duración óptima del tratamiento antibiótico en recién nacidos con sospecha de sepsis neonatal temprana con resultados de hemocultivo negativos.

- Validez, confiabilidad, utilidad y extrapolabilidad de las escalas sumativas para el diagnóstico de sepsis neonatal.

- Asociación entre manifestaciones clínicas en RN con sepsis neonatal.

clínica favorable, se recomienda suspender el tratamiento antibiótico entre los 48 y 72 h. Recomendación fuerte a favor de la intervención basada en opinión de expertos.
En RN con sospecha de infección, con hemocultivos negativos y clínica sospechosa de infección (o sin evolución clínica favorable), se recomienda continuar el tratamiento antibiótico por 7 días. Reco- mendación fuerte a favor de la intervención basada en opinión de expertos.

En caso de pobre respuesta o fracaso terapéutico, debe pasar a segunda línea de antibiótico, según el mapa microbioló-

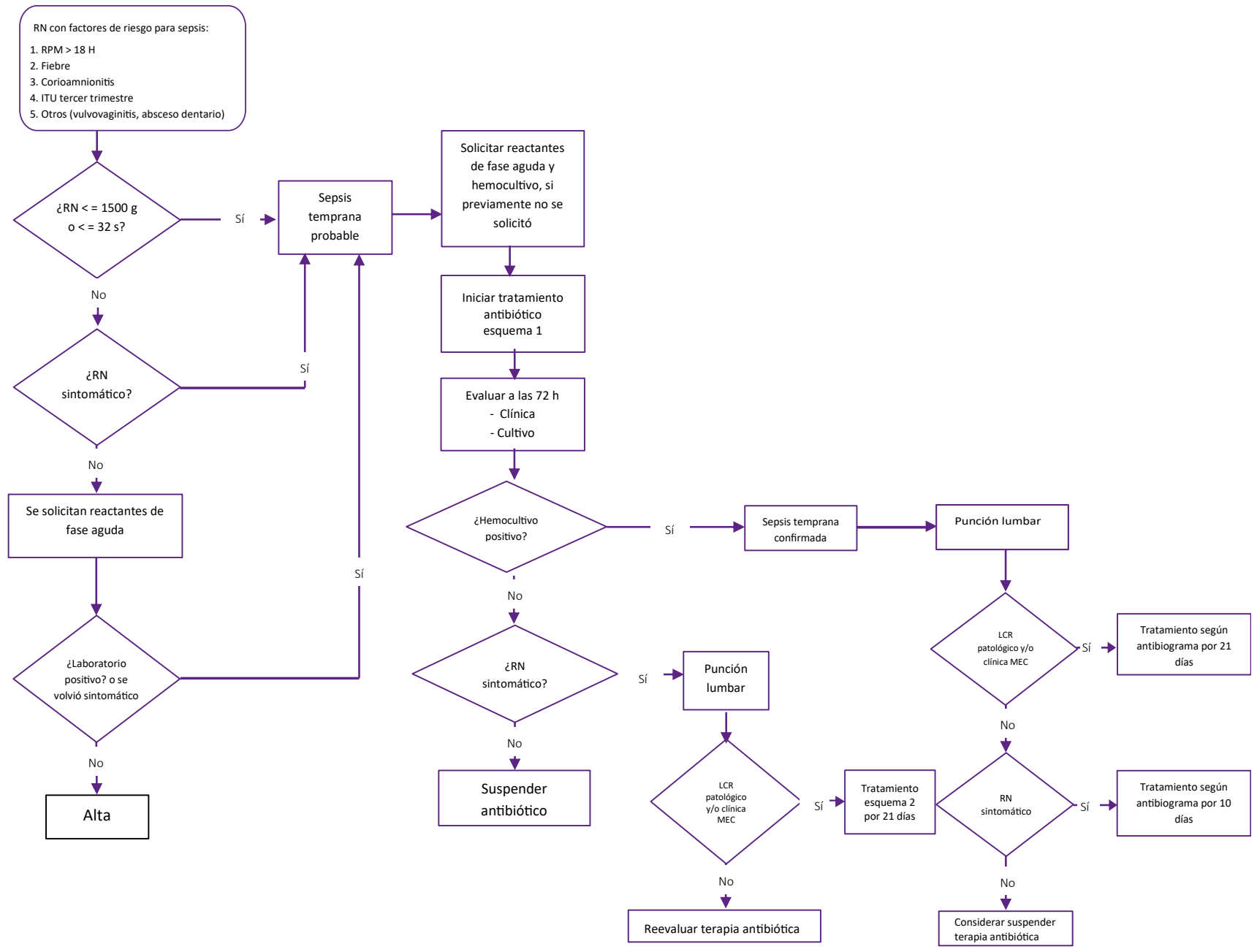

Figura 1. Fluxograma de sepsis neonatal temprana. 


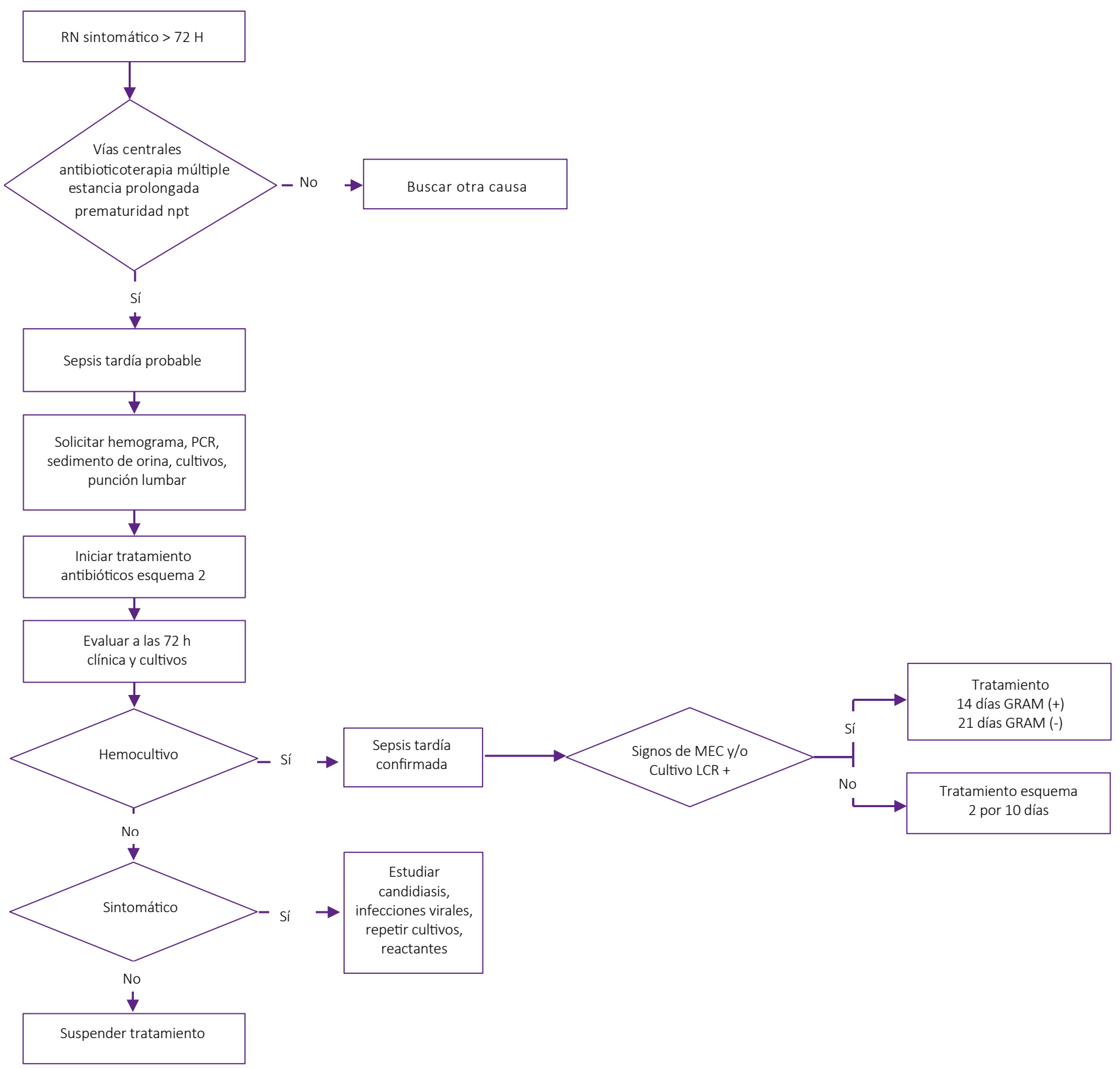

Figura 2. Fluxograma sepsis neonatal tardía.

gico institucional. Recomendación fuerte a favor de la intervención basada en opinión de expertos.

\section{De la evidencia científica a la práctica} clínica

No se encontró evidencia directa que apoye la decisión sobre la duración del tratamiento antibiótico en RN con sospecha de sepsis neonatal. Una GPC de NICE propone, en base a consenso de expertos, mantener el tratamiento antibiótico durante 5 a 7 días ${ }^{(37)}$.
Pregunta 15. En RN con sospecha de infección neonatal con resultado de hemocultivos positivos, ¿cuál es la duración óptima del tratamiento antibiótico?

\section{Recomendación}

En RN con hemocultivos positivos y con evolución clínica favorable, se recomienda completar 7 días de tratamiento antibiótico. Recomendación fuerte a favor de la intervención basada en baja calidad de la evidencia.
En caso de pobre respuesta o fracaso terapéutico, debe pasar a segunda línea de antibiótico, según mapa microbiológico institucional. Recomendación fuerte a favor de la intervención basada en muy baja calidad de la evidencia.

De la evidencia científica a la práctica clínica

Los efectos deseables son grandes para el desarrollo de la recomendación en la práctica clínica comparado con los 
efectos indeseables pequeños. El balance entre los beneficios y riesgos favorecen la intervención. La evidencia de baja calidad no encuentra diferencias entre administrar un régimen de tratamiento de 7 días en RN con sospecha de infección neonatal y el hemocultivo comprobado (38).

Pregunta 16. ¿Cuál es el régimen de tratamiento antibiótico óptimo para la meningitis neonatal?

\section{Recomendación}

Se recomienda dar tratamiento antibiótico por al menos 14 días para meningitis neonatal, evaluando la respuesta clínica, características bioquímicas y microbiológicas del LCR y estudio de imágenes cerebral. Recomendación fuerte a favor de la intervención basada en consenso de expertos.

Se recomienda determinar el régimen antibiótico de acuerdo con el mapa microbiológico de la institución en las meningitis asociadas a sepsis tardía. En las meningitis asociadas a sepsis temprana o adquirida en la comunidad se recomienda iniciar tratamiento con ampicilina y cefotaxima a dosis que logren penetrabilidad en el LCR. Recomendación fuerte a favor de la intervención basada en consenso de expertos.

\section{De la evidencia científica a la práctica clínica}

Los beneficios de dar tratamiento en casos de meningitis neonatal con buena evolución clínica y laboratoriales de 14 días son elevados con la finalidad de evitar recaída del cuadro de meningitis, la cual elevaría el daño neurológico y las secuelas posteriores al mismo ${ }^{(39)}$. Los daños potenciales de esta recomendación serían, uso prolongado de antibióticos con los eventos adversos relacionados a su uso y mayor estancia hospitalaria.

\section{RECOMENDACIONES PARA LA INVESTIGACIÓN}

Durante los diálogos deliberativos y a través del enfoque GRADE se pudo identificar necesidades de investigación que podrán contribuir a la práctica clínica del manejo de la sepsis neonatal en el contexto peruano (Tabla 5).

El GE realizó de modo adicional una revisión de evidencias que incluye otros aspectos como: factores de riesgo asociados a sepsis neonatal, signos y síntomas comunes, complicaciones, medidas de prevención y un listado de los microorganismos bacterianos frecuentes, añadidos a las recomendaciones basadas en evidencia, permitiendo construir dos flujogramas de atención de la sepsis neonatal, como parte de la implementación (Figuras 1 y 2 ).

\section{REFERENCIASBIBLIOGRÁFICAS}

1. Instituto Nacional de Estadistica e Informática. Encuesta Demográfica y de Salud Familiar-ENDES 2014 [Internet]. 2015. Disponible en: https://www. inei.gob.pe/media/MenuRecursivo/publicaciones_digitales/Est/Lib1211/pdf/Libro.pdf

2. Instituto Nacional de Estadística e Informática. Encuesta Demográfica y de Salud Familiar, Perú - 2016 [Internet]. 2017. Disponible en: http://proyectos.inei gob.pe/endes/Informes/Libro_ENDES\%202016.pdf

3. Jeannette Ávila Vargas-Machuca, Mario Tavera Salazar, Marco Carrasco Gamarra, Dirección General de Epidemiologia, Ministerio de Salud. Mortalidad Neonatal en el Perú y sus departamentos, 2011-2012 [Internet]. ASKHA E.I.R.L.; 2013. Disponible en: https://www.unicef.org/peru/ spanish/Mortalidad-Neonatal-en-el-Peru-y-susdepartamentos-2011-2012.pdf

4. Organización Mundial de la Salud. Reducir la mortalidad de los recién nacidos [Internet]. Notas descriptivas. 2018. Disponible en: https://www. who.int/es/news-room/fact-sheets/detail/reducir-lamortalidad-de-los-reci\%C3\%A9n-nacidos

5. Oestergaard MZ, Inoue M, Yoshida S, Mahanan WR, Gore FM, Cousens S, et al. Neonatal mortality levels for 193 countries in 2009 with trends since 1990: a systematic analysis of progress, projections and priorities. PLoS Med. Agosto de 2011; 8(8) e1001080. DOI: https://doi.org/10.1371/journal. pmed.1001080

6. Ministerio de Salud del Perú, Dirección Genera de Salud de las Personas, Estrategia Sanitaria de Salud Sexual y Reproductiva. Guía Técnica: Guias de Práctica Clínica para la atención del Recién Nacido [Internet]. Disponible en: http://bvs.minsa. gob.pe/local/MINSA/1027_DGSP198.pdf

7. Ministerio de Salud del Perú. Documento Técnico Metodología para la elaboración de Guias de Práctica Clinica [Internet]. 2015. Disponible en: http:// www.minsa.gob.pe/dgsp/documentos/Guias/MGPC1.pdf

8. Consorcio AGREE. Instrumento para la evaluación de Guias de Práctica Clínica, AGREE II [Internet]. Disponible en: http://www.guiasalud.es/contenidos/ documentos/Guias_Practica_Clinica/SpanishAGREE-II.pdf
9. Ministerio de salud y protección social, Colombia, IETS. Guía de práctica clínica Recién nacido: sepsis neonatal temprana - 2013 Guia Nº6 [Internet]. 2013. Disponible en: http://gpc.minsalud.gov. co/gpc_sites/Repositorio/Conv_500/GPC_rnsn/ GPC_Completa_Sepsis.pdf

10. Grupo de trabajo GRADE. Manual GRADE (Grading of Recommendations, Assessment, Development and Evaluation), Version en Español 2017 [Internet]. 2017. Disponible en: http://gdt.guidelinedevelopment.org/app/

11. Carmona G, Bonilla C, Huamán K, Reyes N, Hijar G, Caballero P. Utilidad de los diálogos deliberativos para la formulación de recomendaciones de guías de práctica clínica. Rev Perú Med Exp Salud Pública. 2017; 34(4):738-43. DOI: 10.17843/ rpmesp.2017.344.2886

12. Ungerer RLS, Lincetto O, McGuire W, Saloojee $\mathrm{H}$, Gulmezoglu AM. Prophylactic versus selective antibiotics for term newborn infants of mothers with risk factors for neonatal infection. Cochrane Database Syst Rev. 2004;(4):CD003957. DOI: 10.1002/14651858.CD003957.pub2

13. Kuzniewicz MW, Puopolo KM, Fischer A, Walsh EM, Li S, Newman TB, et al. A Quantitative, Risk-Based Approach to the Management of Neonatal EarlyOnset Sepsis. JAMA Pediatr. 2017; 171(4):365-71. DOI: 10.1001/jamapediatrics.2016.4678

14. Agati G, Fusi F, Donzelli GP, Pratesi R. Quantum yield and skin filtering effects on the formation rate of lumirubin. J Photochem Photobiol B. 1993; 18(23):197-203. DOI: 10.1016/1011-1344(93)80063-f

15. Towers CV, Yates A, Zite N, Smith C, Chernicky L, Howard B. Incidence of fever in labor and risk of neonatal sepsis. Am J Obstet Gynecol. 2017; 216(6): 596.e1-596.e5. DOI: 10.1016/j.ajog.2017.02.022

16. Linder N, Fridman E, Makhoul A, Lubin D, Klinger G, Laron-Kenet $\mathrm{T}$, et al. Management of term newborns following maternal intrapartum fever. J Matern-Fetal Neonatal Med off J Eur Assoc Perinat Med Fed Asia Ocean Perinat Soc Int Soc Perinat Obstet. 2013; 26(2):207-10. DOI: 10.3109/14767058.2012.722727

17. Dior UP, Kogan L, Eventov-Friedman S, Gil M, Bahar R, Ergaz Z, et al. Very High Intrapartum Fever in Term Pregnancies and Adverse Obstetric and Neonatal Outcomes. Neonatology. 2016; 109(1):62-8. DOI: 10.1159/000440938

18. Pérez RO, Lona JC, Quiles M, Verdugo MÁ, Ascencio EP, Benitez EA. Sepsis neonatal temprana, incidencia y factores de riesgo asociados en un hospital público del occidente de México. Rev Chil Infectol. 2015; 32(4):387-392.

19. Das A, Shukla S, Rahman N, Gunzler D, Abughali N. Clinical Indicators of Late-Onset Sepsis Workup in Very Low-Birth-Weight Infants in the Neonatal Intensive Care Unit. Am J Perinatal. 2016; 33(9):856-60. DOI: 10.1055/s-0036-1579648

20. Dantas AVVC, Farias LJR, de Paula SJ, Moreira RP, da Silva VM, López MVO, et al. Nursing Diagnosis of Neonatal Jaundice: Study of Clinical Indicators. J Pediatr Nurs. 2017; 39: e6-e10. DOI: 10.1016/j. pedn.2017.12.001

21. Srinivasan L, Harris MC. New technologies for the rapid diagnosis of neonatal sepsis. Curr Opin Pediatr. 2012; 24(2):165-71. DOI: 10.1097/ MOP.0b013e3283504df3

22. Newman TB, Puopolo KM, Wi S, Draper D, Escobar GJ. Interpreting complete blood counts soon after birth in newborns at risk for sepsis. Pediatrics. 2010; 126(5):903-9. DOI: 10.1542/peds.2010-0935 
23. Yang A-P, Liu J, Yue L-H, Wang H-Q, Yang W-J, Yang G-H. Neutrophil CD64 combined with PCT, CRP and WBC improves the sensitivity for the early diagnosis of neonatal sepsis. Clin Chem Lab Med. 2016; 54(2):345-51. DOI: 10.1515/cclm-2015-0277

24. Altunhan $\mathrm{H}$, Annagur $\mathrm{A}$, Ors R, Mehmetoglu Procalcitonin measurement at 24 hours of age may be helpful in the prompt diagnosis of early-onset neonatal sepsis. Int J Infect Dis IJID off Publ Int Soc Infect Dis. 2011; 15(12): e854-858. DOI: 10.1016/j. ijid.2011.09.007

25. Kordek A. Concentrations of procalcitonin and C-reactive protein, white blood cell count, and the immature-to-total neutrophil ratio in the blood of neonates with nosocomial infections: Gram-negative bacilli vs coagulase-negative staphylococci. Eur J Clin Microbiol Infect Dis. 2011; 30(3):455-7. DOI: 10.1007/s10096-010-0956-5

26. Celik HT, Portakal O, Yigit S, Hascelik G, Korkmaz A Yurdakok M. Efficacy of new leukocyte parameters versus serum C-reactive protein, procalcitonin, and interleukin-6 in the diagnosis of neonatal sepsis. Pediatr Int. 2016; 58(2):119-25. DOI: 10.1111/ped.12754

27. Tarai B, Das P, Kumar D, Budhiraja S. Comparative evaluation of paired blood culture (aerobic/aerobic) and single blood culture, along with clinica importance in catheter versus peripheral line at a tertiary care hospital. Indian J Med Microbiol. 2012 30(2):187-92. DOI: 10.4103/0255-0857.96689
28. Berg AS, Inchley CS, Fjaerli HO, Leegaard TM, Lindbaek M, Nakstad B. Clinical features and inflammatory markers in pediatric pneumonia: a prospective study. Eur J Pediatr. 2017; 176(5):629-38. DOI: 10.1007/s00431-017-2887-y

29. Riskin A, Toropine A, Bader D, Hemo M, Srugo I, Kugelman A. Is it justified to include urine cultures in early ( $<72$ hours) neonatal sepsis evaluations of term and late preterm infants? Am J Perinatol. 2013; 30(6):499-504. DOI: 10.1055/s-0032-1329180

30. Ray B, Mangalore J, Harikumar C, Tuladhar A. Is lumbar puncture necessary for evaluation of early neonatal sepsis?. Arch Dis Child. 2006; 91(12):10331035. DOI: 10.1136/adc.2006.105106

31. Visser VE, Hall RT. Lumbar puncture in the evaluation of suspected neonatal sepsis. J Pediatr. 1980:96(6):1063-7. DOI: 10.1016/s00223476(80)80643-3

32. Eldadah M, Frenkel LD, Hiatt IM, Hegyi T. Evaluation of routine lumbar punctures in newborn infants with respiratory distress syndrome. Pediatr Infect Dis J. 1987; 6(3):243-6. DOI: 10.1097/00006454198703000-00005

33. Hendricks-Munoz KD, Shapiro DL. The role of the lumbar puncture in the admission sepsis evaluation of the premature infant. J Perinatol. 1990; 10(1):60-4.

34. Weiss MG, Ionides SP, Anderson CL. Meningitis in premature infants with respiratory distress: role of admission lumbar puncture. J Pediatr. 1991; 119(6):973-5. DOI: 10.1016/s00223476(05)83059-8

35. Ajayi OA, Mokuolu OA. Evaluation of neonates with risk for infection/suspected sepsis: is routine lumbar puncture necessary in the first 72 hours of life?. Trop Med Int Health. 1997; 2(3):284-8. DOI: 10.1046/j.1365-3156.1997.d01-270.x

36. Metsvaht T, IImoja M-L, Parm U, Maipuu L, Merila M, Lutsar I. Comparison of ampicillin plus gentamicin vs. penicillin plus gentamicin in empiric treatment of neonates at risk of early onset sepsis. Acta Paediatr. 2010; 99(5): 665-72. DOI: 10.1111/j.16512227.2010.01687.x

37. National Institute for Health and Care Excellence (NICE). Sepsis: recognition, diagnosis and early management NICE guideline [NG51] [Internet]. 2017. Disponible en: https://www.nice.org.uk/guidance/ ng51

38. Chowdhary G, Dutta S, Narang A. Randomized controlled trial of 7-Day vs. 14-Day antibiotics for neonatal sepsis. J Trop Pediatr. 2006; 52(6):427-32. DOI: 10.1093/tropej/fml054

39. Mathur NB, Kharod P, Kumar S. Evaluation of duration of antibiotic therapy in neonatal bacterial meningitis: a randomized controlled trial. J Trop Pediatr. 2015; 61(2):119-25. DOI: 10.1093/tropej/ fmv002 